\title{
The expression of platelet-derived growth factor, epidermal growth factor, and insulin-like growth factor-II in patients with polycystic ovary syndrome and its correlation with pregnancy outcomes
}

\author{
Jiefan Gao ${ }^{1}$, Yangyang Song ${ }^{1}$, Xiao Huang ${ }^{1}$, Donghui Wang ${ }^{1}$, Huilan Wang ${ }^{2}$ \\ ${ }^{1}$ Department of Reproductive Medicine, Cangzhou Central Hospital, Cangzhou, China; ${ }^{2}$ Department of Gynecology, the Second Hospital of Hebei \\ Medical University, Shijiazhuang, China \\ Contributions: (I) Conception and design: J Gao, H Wang; (II) Administrative support: Y Song, X Huang; (III) Provision of study materials or patients: \\ J Gao, Y Song, X Huang, D Wang; (IV) Collection and assembly of data: All authors; (V) Data analysis and interpretation: J Gao, D Wang, H Wang; \\ (VI) Manuscript writing: All authors; (VII) Final approval of manuscript: All authors. \\ Correspondence to: Huilan Wang. Department of Reproductive Medicine, Cangzhou Central Hospital, 16 Xinhua West Road, Cangzhou 061000, \\ China. Email: czzxyygj@@126.com.
}

Background Polycystic ovary syndrome (PCOS) is a common endocrine and metabolic disorder with complex pathogenesis. This study aimed to analyze the expression of platelet-derived growth factor (PDGF), epidermal growth factor (EGF), and insulin-like growth factor-II (IGF-II) in patients with PCOS and its correlation with pregnancy outcomes.

Methods: A total of 104 PCOS patients admitted to the Cangzhou Central Hospital from January 2015 to February 2018 were selected as the PCOS group, and 92 healthy pregnant women who received health examinations in the hospital during the same period were selected as the control group. Levels of PDGF, EGF, and IGF-II in serum were detected. The expression of PDGF, EGF, and IGF-II in different populations were compared. Age at pregnancy, body mass index (BMI), waist-to-hip ratio before pregnancy, parity, family history of hypertension, family history of diabetes, and serological indicators of pregnant women in the PCOS group were collected, such as follicle stimulating hormone (FSH), luteinizing hormone (LH), estradiol (E2), fasting insulin (INS), and free testosterone index (FTI). Multivariate logistic regression was used to analyze the risk factors that affect the pregnancy outcomes of PCOS patients.

Results: The expression levels of PDGF, EGF, and IGF-II in the PCOS group were significantly higher than those in the control group $(\mathrm{P}<0.05)$. Among 76 pregnant PCOS patients, 34 cases had adverse pregnancy outcomes and 42 did not. Age at pregnancy, BMI before pregnancy, waist-to-hip ratio before pregnancy, LH, INS, FTI, PDGF, EGF, and IGF-II expression levels were positively correlated with the pregnancy outcome of PCOS patients $(\mathrm{P}<0.05)$. The BMI before pregnancy, waist-to-hip ratio before pregnancy, INS, FTI, and expression levels of PDGF, EGF, and IGF-II were revealed as independent risk factors that affect the pregnancy outcomes of PCOS patients $(\mathrm{P}<0.05)$.

Conclusions: The expression levels of PDGF, EGF, and IGF-II are high in PCOS patients. The BMI, waist-to-hip ratio before pregnancy, INS, FTI, and the expression levels of PDGF, EGF, IGF-II are independent risk factors that affect the pregnancy outcomes of PCOS patients. Corresponding measures should be actively taken for PCOS patients with high-risk factors to improve both maternal and infant outcomes.

Keywords: Platelet-derived growth factor (PDGF); epidermal growth factor (EGF); insulin-like growth factor-II (IGF-II); polycystic ovary syndrome (PCOS); pregnancy outcomes 
Submitted Apr 07, 2021. Accepted for publication May 21, 2021.

doi: 10.21037/apm-21-1003

View this article at: http://dx.doi.org/10.21037/apm-21-1003

\section{Introduction}

Polycystic ovary syndrome (PCOS) is a common endocrine and metabolic disorder among women of childbearing age. It is characterized by chronic anovulation and hyperandrogenemia, with the main clinical manifestations of irregular menstrual cycles, infertility, hirsutism, and (or) acne $(1,2)$. However, the pathogenesis of PCOS is not clear and is thought to be related to genetic and immune factors. Studies have shown that without effective treatment, PCOS will seriously affect the quality of life, reproductive function, and long-term health of women, so early diagnosis and timely assessment of the disease development are of great significance (3).

Platelet-derived growth factor (PDGF) and epidermal growth factor (EGF) play an important role in regulating egg cell growth and embryonic development. In recent years, the relationship between the two indicators and PCOS has become a hot topic among researchers in China and the world over (4). In addition, many studies have found that insulin-like growth factor-II (IGF-II) is abnormally expressed in tumors of female reproductive system. However, there are few reports about the expression of PDGF, EGF and IGFII in PCOS and their relationship with pregnancy outcome in China (5). In this study, we analyzed the expression of PDGF, EGF, and IGF-II in PCOS patients and their correlation with pregnancy outcomes, to explore the role of the three indicators in the occurrence and development of PCOS. We present the following article in accordance with the STROBE reporting checklist (available at http://dx.doi. org/10.21037/apm-21-1003).

\section{Methods}

\section{General information}

A total of 104 PCOS patients admitted to our hospital from January 2015 to February 2018 were recruited to the PCOS group; they were aged 22-65 years old, with an average age of $(41.03 \pm 9.85)$ years. In addition, 92 pregnant women who received health examinations in our hospital during the same period and had normal results were selected as the control group, with an average age of $(41.25 \pm 9.86)$ years.
The difference between the general data of the PCOS group and the control group were not statistically significant and were comparable $(\mathrm{P}>0.05)$.

The inclusion criteria were as follows: (I) patients met the diagnostic criteria of PCOS (6): (i) occasional ovulation and (or) anovulation; (ii) clinical and (or) biochemical indicators suggested hyperandrogenism, excluding other possible pathogenic factors, such as congenital adrenal hyperplasia, androgen-secreting tumors, or Cushing syndrome; (iii) polycystic changes of the ovary: B-ultrasound examination revealed more than 12 follicles with diameters of $2-9 \mathrm{~mm}$ and (or) an increase in the volume of the ovary $>10 \mathrm{~mL}$; PCOS was diagnosed when 2 of the criteria were met; (II) patients who were willing to give birth; (III) patients who were aware of and signed the relevant agreements.

The exclusion criteria were as follows: (I) patients with genital deformities; (II) infertility caused by other reasons; (III) patients with mental or communication disorders; (IV) patients had taken hormone drugs such as contraceptives, hypoglycemic, or hypotensive drugs in the previous 3 months; (V) patients combined with endometriosis, ovarian tumors, and other organic diseases that affect pregnancy; (VI) comorbidity of severe liver and kidney dysfunction, cardiovascular, and cerebrovascular diseases; (VII) patients who had experienced a major infection in the past 3 months. All procedures performed in this study involving human participants were in accordance with the Declaration of Helsinki (as revised in 2013). This study was approved by the Ethics Committee of Cangzhou Central Hospital [2271]. Informed consent was taken from all the patients.

\section{Research methods}

Along with comparing the expression of PDGF, EGF, and IGF-II between the PCOS and control groups, clinical data were collected including age, pre-pregnancy body mass index (BMI), pre-pregnancy waist-to-hip ratio (waist/ hip circumference), parity, family history of hypertension, family history of diabetes, and serological indicators, including follicle stimulating hormone (FSH), luteinizing hormone (LH), estradiol (E2), fasting insulin (INS), and free testosterone index (FTI). 
Table 1 Comparison of the expression levels of PDGF, EGF, and IGF-II between the two groups $(\bar{x} \pm \mathrm{s})$

\begin{tabular}{lcccc}
\hline Group & Case & PDGF $(\mathrm{ng} / \mathrm{mL})$ & EGF $(\mathrm{ng} / \mathrm{mL})$ & IGF-II $(\mathrm{ng} / \mathrm{L})$ \\
\hline PCOS group & 104 & $3,154.66 \pm 214.75$ & $105.96 \pm 25.74$ & $3.02 \pm 0.69$ \\
Control group & 92 & $2,017.25 \pm 147.69$ & $75.99 \pm 13.01$ & $0.43 \pm 0.11$ \\
$t$ & - & 36.752 & 10.084 & 35.595 \\
P value & - & $<0.001$ & $<0.001$ & $<0.001$ \\
\hline
\end{tabular}

PDGF, platelet-derived growth factor; EGF, epidermal growth factor; IGF-II, insulin-like growth factor-II; PCOS, polycystic ovary syndrome.

\section{Detection methods of PDGF, EGF, and IGF-II}

For the detection, $5 \mathrm{~mL}$ of cubital vein blood was drawn from all participants, and the supernatant of the blood was collected for further test after centrifugation at 3,000 r/min for $30 \mathrm{~min}$. An enzyme-linked immunosorbent assay (ELISA) kit was used to detect the levels of PDGF and EGF in serum (Sigma-Aldrich, St. Louis, MO, USA). The levels were measured by detecting absorbance at $410 \mathrm{~nm}$ on an ELISA instrument, with a standard line drawn. The serum IGF-II level was determined using Immulite 2000 automatic chemiluminescence analyzer kit (Siemens Medical Diagnostic Products, Shanghai, China) and the supporting reagents, according to the instructions.

\section{Follow-up methods}

After treatment, 76 of the 104 PCOS participants became pregnant during the study period. Pregnant women in both groups underwent routine obstetric examinations. The health of pregnant women and fetuses were closely monitored, and the final pregnancy outcomes of the PCOS group and the control group were recorded. The diagnostic criteria for adverse pregnancy outcomes were as follows: a pregnancy outcome was deemed adverse when hospitalization was required due to pregnancy complications such as threatened miscarriage, preeclampsia, threatened premature delivery, poly- or oligohydramnios, prenatal hemorrhage, fetal distress, fetal growth restriction, stillbirth, or severe fetal malformations, and other pregnancy complications (7).

\section{Statistical methods}

Data in this study were analyzed using the statistical software SPSS 22.0 (IBM Corp., Armonk, NY, USA). The measurement data were described as mean \pm standard deviation $(\bar{x} \pm s)$, and analyzed by $t$-test. The count data were expressed as pass rate or composition ratio, and analyzed by chi-squared $\left(\chi^{2}\right)$ test. Multiple logistic regression analysis was used to analyze risk factors that affected the pregnancy outcomes of PCOS patients. Results with $\mathrm{P}<0.05$ were considered statistically significant.

\section{Results}

\section{Comparison of the expression levels of PDGF, EGF, and IGF-II between the two groups}

The expression levels of PDGF, EGF, and IGF-II in the PCOS group were significantly higher than those in the control group $(\mathrm{P}<0.05$, Table 1$)$.

\section{Pregnancy outcomes of 76 participants in the PCOS group}

A total of 34 participants had adverse pregnancy outcomes among the 76 pregnant PCOS participants. They were regarded as the adverse pregnancy outcome group $(n=34)$, accounting for $44.74 \%$, including 16 cases of threatened abortion, 6 cases of preeclampsia, 5 cases of threatened preterm delivery, 2 cases of polyhydramnios, 2 cases of oligohydramnios, 1 case of prenatal hemorrhage, 1 case of fetal distress, and 1 case of stillbirth. The remaining 42 cases with no adverse pregnancy outcomes were classified as the no adverse pregnancy outcome group $(n=42)$.

\section{Univariate analysis of the pregnancy outcome of PCOS patients}

Factors including age, pre-pregnancy BMI, pre-pregnancy waist-to-hip ratio, LH, INS, FTI, and PDGF, EGF, and IGF-II expression levels were positively correlated with pregnancy outcomes in PCOS patients, while parity, family history of hypertension, family history of diabetes, FSH, and E2 were not related to the pregnancy outcomes of PCOS patients $(\mathrm{P}<0.05$, Table 2). 
Table 2 Univariate analysis of pregnancy outcomes in PCOS patients

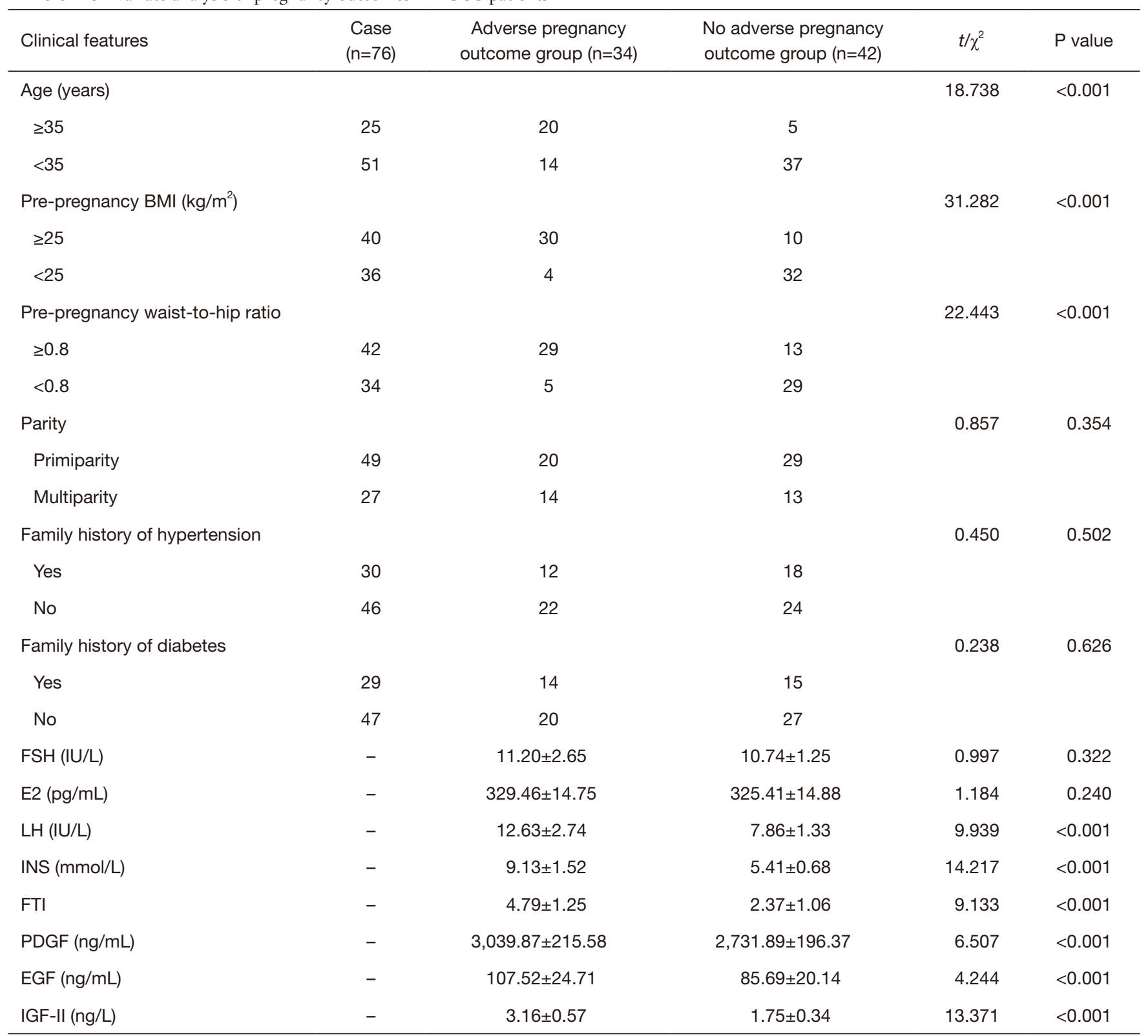

PCOS, polycystic ovary syndrome; BMI, body mass index; FSH, follicle stimulating hormone; E2, estradiol; LH, luteinizing hormone; INS, fasting insulin; FTI, free testosterone index; PDGF, platelet-derived growth factor; EGF, epidermal growth factor; IGF-II, insulin-like growth factor-II.

\section{Analysis of multiple factors affecting pregnancy outcomes of PCOS patients}

The significance and assignment of the factors to be analyzed are shown in Table 3. After unconditional multivariate logistic regression analysis, it was shown that pre-pregnancy BMI, pre-pregnancy waist-to-hip ratio, INS, FTI, PDGF, EGF, and IGF-II expression levels were independent risk factors affecting the pregnancy outcomes of PCOS patients $(\mathrm{P}<0.05$, Table 4$)$.

\section{Discussion}

The pathogenesis of PCOS is complex; it can be induced by multiple factors, alone or in combination, such as heredity, 
immunity, and environmental (8). With the continuous development of clinical assisted reproductive technology, most patients with PCOS can now successfully achieve pregnancy. However, in clinical practice, PCOS itself can increase the occurrence of adverse pregnancy outcomes such as miscarriage and low birth weight infants. The woman's poor vascular status may induce insufficient placental function, and early exposure to male hormones may lead to the occurrence of intrauterine growth restriction in the

Table 3 Significance and assignment of factors to be analyzed

\begin{tabular}{lll}
\hline Factor & \multicolumn{1}{c}{$\begin{array}{c}\text { Representative } \\
\text { significance }\end{array}$} & \multicolumn{1}{c}{ Assignment } \\
\hline $\mathrm{X}_{1}$ & Age & $0=<35 ; 1=\geq 35$ \\
$\mathrm{X}_{2}$ & Pre-pregnancy BMI & $0=<25 ; 1=\geq 25$ \\
$\mathrm{X}_{3}$ & Pre-pregnancy waist-to- & $0=<0.8 ; 1=\geq 0.8$ \\
& hip ratio & $0=$ normal; $1=$ increased \\
$\mathrm{X}_{4}$ & LH & $0=$ normal; $1=$ increased \\
$\mathrm{X}_{5}$ & INS & $0=$ normal; $1=$ increased \\
$\mathrm{X}_{6}$ & FTI & $0=$ normal; $1=$ increased \\
$\mathrm{X}_{7}$ & PDGF & $0=$ normal; $1=$ increased \\
$\mathrm{X}_{8}$ & EGF & $0=$ normal; $1=$ increased \\
$\mathrm{X}_{9}$ & IGF-II & \\
\hline
\end{tabular}

BMI, body mass index; LH, luteinizing hormone; INS, fasting insulin; FTI, free testosterone index; PDGF, platelet-derived growth factor; EGF, epidermal growth factor; IGF-II, insulin-like growth factor-II. fetus, thereby impacting by varying degrees on the health of the offspring $(9,10)$. Large volumes of literature from China and around the world have indicated that it is clinically valuable to improve the pregnancy outcomes of PCOS patients through the exploration of the relevant factors that affect such outcomes for PCOS patients and provision of targeted intervention measures (11).

The results of this study showed that 34 cases among 76 pregnant PCOS patients had adverse pregnancy outcomes, accounting for $44.74 \%$, basically consistent with previous research results (12). Patients with PCOS have endocrine and metabolic disorders, and most patients are accompanied by varying degrees of insulin cell function defects, peripheral tissue insulin resistance, and dyslipidemia, which increases the risk of pregnancy-induced hypertension and gestational diabetes to a certain extent, leading to the poor pregnancy outcomes of PCOS patients (13). The results of this study showed that the expression levels of INS and FTI in the adverse pregnancy outcome group were higher than those in the no adverse pregnancy outcome group $(\mathrm{P}<0.05)$, indicating that abnormal expression of INS and FTI were independent risk factors affecting the pregnancy outcomes of PCOS patients. In addition, this study also showed that pre-pregnancy BMI and pre-pregnancy waist-to-hip ratio were independent risk factors that affect the pregnancy outcome of PCOS patients $(\mathrm{P}<0.05)$, because obesity or overweight may cause placental inflammation in the body, affect placental function, and cause adverse outcomes.

As a kind of cationic glycoprotein, PDGF is a major mitogen and chemotactic agent in the body (14). In the

Table 4 Multivariate analysis of pregnancy outcomes in PCOS patients

\begin{tabular}{lccccc}
\hline Factor & Regression coefficient & SEM & Wald $\chi^{2}$ & P value & OR (95\% Cl) \\
\hline Age ( $\geq 35$ vs. $<35)$ & 0.975 & 0.846 & 3.459 & 0.445 & $2.651(0.505-13.918)$ \\
Pre-pregnancy BMI ( $\geq 25$ vs. $<25)$ & 0.932 & 0.151 & 4.184 & $<0.001$ & $2.540(1.889-3.414)$ \\
Pre-pregnancy waist-to-hip ratio ( $\geq 0.8$ vs. $<0.8)$ & 0.987 & 0.147 & 3.157 & $<0.001$ & $2.68(2.011-3.579)$ \\
LH (increased vs. normal) & 0.897 & 0.785 & 4.595 & 0.321 & $2.452(0.526-11.423)$ \\
INS (increased vs. normal) & 1.056 & 0.423 & 3.480 & $<0.001$ & $2.875(1.255-6.587)$ \\
FTI (increased vs. normal) & 1.745 & 0.374 & 4.053 & $<0.001$ & $5.726(2.751-11.918)$ \\
PDGF (increased vs. normal) & 0.844 & 0.156 & 4.274 & $<0.001$ & $2.326(1.713-3.157)$ \\
EGF (increased vs. normal) & 0.716 & 0.113 & 4.506 & $<0.001$ & $2.046(1.640-2.554)$ \\
IGF-II (increased vs. normal) & 0.973 & 0.187 & 4.847 & $<0.001$ & $2.646(1.834-3.817)$ \\
\hline
\end{tabular}

PCOS, polycystic ovary syndrome; SEM, standard error of mean; OR, odds ratio; Cl, confidence interval; LH, luteinizing hormone; INS, fasting insulin; FTI, free testosterone index; PDGF, platelet-derived growth factor; EGF, epidermal growth factor; IGF-II, insulin-like growth factor-II. 
ovarian area, PDGF participates in regulation of the ovary gland in an autocrine and paracrine manner, which has the pathological effect of promoting excessive growth of related tissue cells. Studies have shown that PDGF can stimulate the proliferation of ovarian alveolar cells, and inhibit the secretion of progesterone and androstenedione, therefore playing an important role in the development and growth of follicles in the body (15). Another growth factor, EGF, is a powerful mitogen like PDGF, which can promote cell proliferation and differentiation, and exert a variety of biological effects. Under normal circumstances, the local cytokines of the ovary maintain a dynamic balance, and there is a small amount of PDGF and EGF in the body. When the local androgen of the ovary rises to a certain threshold, the body will activate the PDGF secretion system and enhance the activity of aromatase by modifying the FSHcAMP pathway to promote the conversion of androgens to estrogen (16). When estrogen increases to a certain threshold, the increase of EGF secretion is promoted, and the aromatase activity is inhibited through the FSHcAMP pathway to reduce androgen conversion (17). Therefore, studies have indicated that PDGF and EGF may play an important role in the occurrence and development of ovarian diseases. The results of this study showed that the levels of PDGF and EGF in the PCOS group were higher than those in the control group, and there were also high expression of PDGF and EGF in patients with adverse pregnancy outcomes, confirming that the levels of PDGF and EGF play an important role in the occurrence of PCOS.

IGF is a class of polypeptides with structures similar to insulin, including IGF-I, IGF-II, and IGF-IR. Among them, IGF-II is a single-chain weakly acidic polypeptide composed of 67 amino acids, which can stimulate the aromatization of progesterone in the ovary, and then stimulate the secretion of progesterone and androgen and promote the production of corpus luteum (18). According to Kreimer et al., IGF-II in follicular fluid of PCOS patients was significantly higher than that of non-PCOS patients, and the concentration of IGF-II in granulosa cells was also higher than that of non-PCOS patients, suggesting its certain relationship with the synchronous development of multiple follicles in the super-ovulation cycle (19). The results of this study showed that IGF-II was highly expressed in PCOS patients, suggesting that IGF-II is closely related to the occurrence of PCOS. In addition, studies have shown that IGF-II is abnormally expressed in PCOS patients, which can affect the development and remodeling of the uterine-placental vascular bed, leading to placental ischemia and hypoxia, placental dysplasia, and ultimately adverse pregnancy outcomes. Therefore, the results of this study indicated that IGF-II expression level is an independent risk factor that affects the pregnancy outcomes of women with PCOS (20). However, the sample size of this study is relatively small, and the results may be biased to some extent. In addition, the fertility and pregnancy outcome of PCOS patients after treatment, as well as the changes in PDGF, EGF and IGF-I expression have not been analyzed. Therefore, in the further investigation, the sample size needed to be expanded.

In summary, PDGF, EGF, and IGF-II are highly expressed in PCOS patients. Factors such as prepregnancy BMI, pre-pregnancy waist-to-hip ratio (waist/ hip circumference), INS, FTI, and the expression levels of PDGF, EGF, and IGF-II are independent risk factors that affect the pregnancy outcomes of women with PCOS. Therefore, for PCOS patients with high risk factors, the treatment regimen should be corrected to improve the clinical maternal and infant outcomes.

\section{Acknowledgments}

Funding: None.

\section{Footnote}

Reporting Checklist: The authors have completed the STROBE reporting checklist. Available at http://dx.doi. org/10.21037/apm-21-1003

Data sharing statement: Available at http://dx.doi. org/10.21037/apm-21-1003

Conflicts of Interest: All authors have completed the ICMJE uniform disclosure form (available at http://dx.doi. org/10.21037/apm-21-1003). The authors have no conflicts of interest to declare.

Ethical Statement: The authors are accountable for all aspects of the work in ensuring that questions related to the accuracy or integrity of any part of the work are appropriately investigated and resolved. This study was approved by the Ethics Committee of Cangzhou Central Hospital [2271]. All procedures performed in this study involving human participants were in accordance with the Declaration of Helsinki (as revised in 2013). Informed consent was taken from all the patients. 
Open Access Statement: This is an Open Access article distributed in accordance with the Creative Commons Attribution-NonCommercial-NoDerivs 4.0 International License (CC BY-NC-ND 4.0), which permits the noncommercial replication and distribution of the article with the strict proviso that no changes or edits are made and the original work is properly cited (including links to both the formal publication through the relevant DOI and the license). See: https://creativecommons.org/licenses/by-nc-nd/4.0/.

\section{References}

1. Teede HJ, Misso ML, Costello MF, et al.

Recommendations from the international evidence-based guideline for the assessment and management of polycystic ovary syndrome. Hum Reprod 2018;33:1602-18. Erratum in: Hum Reprod 2019;34:388.

2. Polak K, Czyzyk A, Simoncini T, et al. New markers of insulin resistance in polycystic ovary syndrome. J Endocrinol Invest 2017;40:1-8.

3. Pierce GF, Mustoe TA, Senior RM, et al. In vivo incisional wound healing augmented by platelet-derived growth factor and recombinant c-sis gene homodimeric proteins. J Exp Med 1988;167:974-87.

4. Li D, Hua Y, Jiang L, et al. Cyclic Guanosine Monophosphate (cGMP)-Dependent Protein Kinase II Blocks Epidermal Growth Factor (EGF)/Epidermal Growth Factor Receptor (EGFR)-Induced Biological Effects on Osteosarcoma Cells. Med Sci Monit 2018;24:1997-2002.

5. Harris HR, Babic A, Webb PM, et al. Polycystic ovary syndrome, oligomenorrhea, and risk of ovarian cancer histotypes: evidence from the Ovarian Cancer Association Consortium. Cancer Epidemiol Biomarkers Prev 2018;27:174-82.

6. Rijlaarsdam J, Cecil CA, Walton E, et al. Prenatal unhealthy diet, insulin-like growth factor 2 gene (IGF2) methylation, and attention deficit hyperactivity disorder symptoms in youth with early-onset conduct problems. J Child Psychol Psychiatry 2017;58:19-27.

7. Wong RCB, Pera MF, Pébay A. Maintenance of Human Embryonic Stem Cells by Sphingosine-1-Phosphate and Platelet-Derived Growth Factor. Methods Mol Biol 2018;1697:133-40.

8. Ratajczak MZ, Bartke A, Darzynkiewicz Z. Prolonged growth hormone/insulin/insulin-like growth factor nutrient response signaling pathway as a silent killer of stem cells and a culprit in aging. Stem Cell Rev Rep
2017;13:443-53.

9. Demmer J, Hill DF, Petersen GB. Characterization of two sheep insulin-like growth factor II cDNAs with different 5 '-untranslated regions. Biochim Biophys Acta 1993;1173:79-80.

10. Wild RA, Carmina E, Diamanti-Kandarakis E, et al. Assessment of cardiovascular risk and prevention of cardiovascular disease in women with the polycystic ovary syndrome: a consensus statement by the Androgen Excess and Polycystic Ovary Syndrome (AE-PCOS) Society. J Clin Endocrinol Metab 2010;95:2038-49.

11. Al-Jefout M, Alnawaiseh N, Al-Qtaitat A. Insulin resistance and obesity among infertile women with different polycystic ovary syndrome phenotypes. Sci Rep 2017;7:5339.

12. Pau CT, Mosbruger T, Saxena R, et al. Phenotype and tissue expression as a function of genetic risk in polycystic ovary syndrome. PLoS One 2017;12:e0168870.

13. Westermark B, Siegbahn A, Heldin CH, et al. B-type receptor for platelet-derived growth factor mediates a chemotactic response by means of ligand-induced activation of the receptor protein-tyrosine kinase. Proc Natl Acad Sci U S A 1990;87:128-32.

14. Blakeley DM, Corps AN, Brown KD. Bombesin and platelet-derived growth factor stimulate formation of inositol phosphates and Ca2+ mobilization in Swiss 3T3 cells by different mechanisms. Biochem J 1989;258:177-85.

15. Rees-Jones RW, Goldfarb M, Goff SP. Abelson murine leukemia virus induces platelet-derived growth factorindependent fibroblast growth: correlation with kinase activity and dissociation from full morphologic transformation. Mol Cell Biol 1989;9:278-87.

16. Kang MK, Mao W, Lee JB, et al. Epidermal growth factor (EGF) fragment-guided anticancer theranostic particles for $\mathrm{pH}$-responsive release of doxorubicin. Int J Pharm 2017;519:104-12.

17. Montoya A, Yepes L, Bedoya A, et al. Transforming growth factor beta (TGFß1) and epidermal growth factor (EGF) as biomarkers of leishmania (V) braziliensis infection and early therapeutic response in cutaneous leishmaniasis: studies in hamsters. Front Cell Infect Microbiol 2018;8:350.

18. Caldwell ASL, Edwards MC, Desai R, et al. Neuroendocrine androgen action is a key extraovarian mediator in the development of polycystic ovary syndrome. Proc Natl Acad Sci U S A 2017;114:E3334-43.

19. Kreimer S. Plasma insulin-like growth factor binding protein 2 is associated with cognitive outcomes. Neurology 
Today 2019;19:18-9.

20. Mancarella C, Caldoni G, Ribolsi I, et al. Insulin-like growth factor $2 \mathrm{mRNA}$-binding protein 3 modulates aggressiveness of Ewing sarcoma by regulating the

Cite this article as: Gao J, Song Y, Huang X, Wang D, Wang H. The expression of platelet-derived growth factor, epidermal growth factor, and insulin-like growth factor-II in patients with polycystic ovary syndrome and its correlation with pregnancy outcomes. Ann Palliat Med 2021;10(5):5671-5678. doi: 10.21037/apm-21-1003
CD164-CXCR4 axis. Front Oncol 2020;10:994.

(English Language Editor: J. Jones) 